\title{
Exploring the Psychological Antecedent Factors of the Transition to Secondary Progressive Multiple Sclerosis: A Qualitative Study
}

\author{
Maryam Bidadian', Seyed Kazem Rasoolzadeh Tabatabaei ${ }^{*}$, Abdorreza Naser Moghadasi², Fazlollah Ahmadi ${ }^{3}$ \\ ${ }^{1}$ Department of Psychology, Faculty of Human Sciences, Tarbiat Modares University, Tehran, Iran \\ ${ }^{2}$ Department of Neurological Diseases, Multiple Sclerosis Research Center, Tehran University of Medical Sciences, Tehran, Iran \\ ${ }^{3}$ Department of Nursing, Faculty of Medical Sciences, Tarbiat Modares University, Tehran, Iran
}

\begin{tabular}{|ccc}
\hline & Article Info: & \\
Received: 11 Nov 2019 & Revised: 13 Jan 2020 & Accepted: 12 Feb 2020
\end{tabular}

\section{A BSTRACT}

Introduction: One of the most common concerns of patients who suffer from multiple sclerosis (MS) is to delay the beginning of the secondary progressive phase. Psychological factors play a role in the exacerbation or recurrence of symptoms of the disease. The current study aimed to explore the psychological antecedent factors of the transition to secondary progressive MS. Materials and Methods: In this qualitative study, 13 participants who were suffering from secondary progressive MS selected by the convenience sampling method in Sina hospital, Tehran, Iran. The current study was conducted based on a thematic analysis approach. Data were collected using a semi-structured interview. Results: The main theme, "Fundamental Mechanisms", which were included two sub-themes, "Core Beliefs" and "Personality Traits" and 10 concepts. Fundamental mechanisms are an abstract concept and this theme can bring the categories together to explain the whole results. Core beliefs sub-theme was included believe in being worthless and unlovable, believe in chance and fate, believe in a dangerous world, believe in being helpless, and self-sacrifice schema. Besides, the personality traits sub-theme was included self-devaluation, dependent personality, narcissistic personality, impulsivity, and intolerance of ambiguity. Conclusion: Outcomes revealed antecedent factors of the transition to secondary progressive MS is based on core beliefs. Furthermore, personality traits are complex, inclusive, and multi-dimensional factors.

\section{Key words:}

1. Multiple Sclerosis

2. Patients

3. Personality

*Corresponding Author: Seyed Kazem Rasoolzadeh Tabatabaei

E-mail: rasoolza@modares.ac.ir 


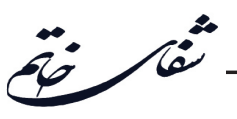

\section{بررسى عوامل روانشناختى زمينهساز در ورود بيماران مبتلا به ام اس به مرحلة بيشروندهُ ثانويه: يك مطالعة كيفى مينى}

مريم بيداديان'، سيد كاظم رسولزاده طباطبايى"'، عبدالرضا ناصر مقدسى'، فضلاله احمدى

'كروه روانشناسى، دانشكده علوم انسانى، دانشكاه تربيت مدرس، تهران، ايران

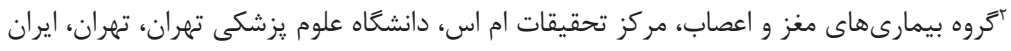

"كروه يرستارى، دانشكده علوم يزشكى، دانشَاه تربيت مدرس، تهران، ايران

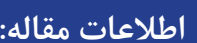

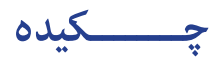

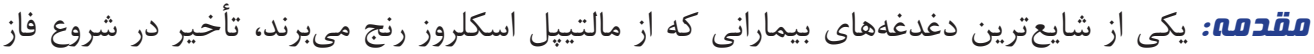

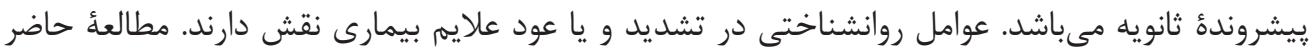

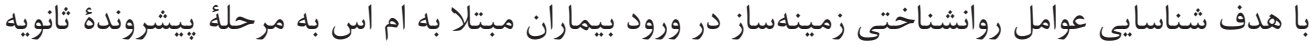

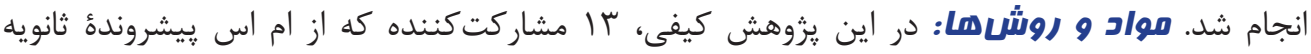

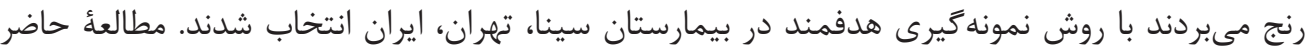

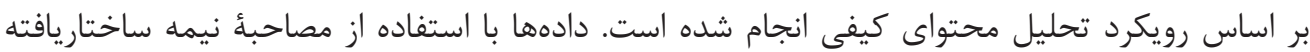

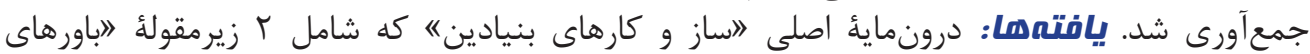

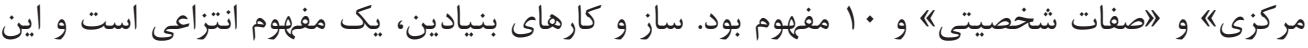

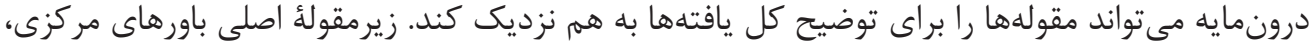

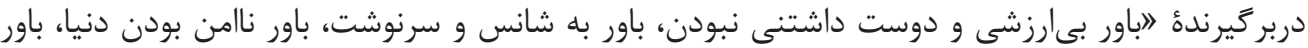

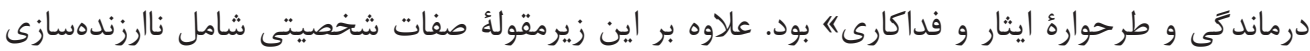

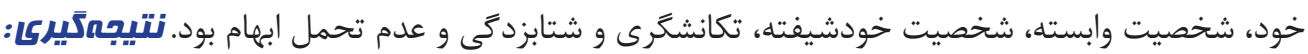

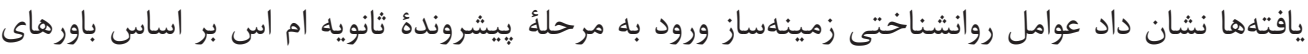

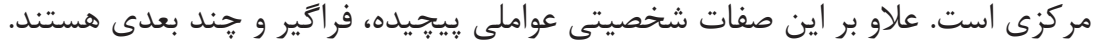

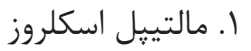

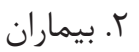

r. ش. شخصيت

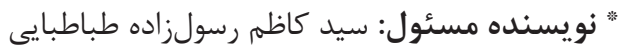
آدرس الكترونيكى: rasoolza@modares.ac.ir 


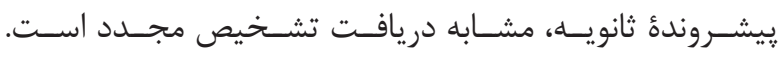

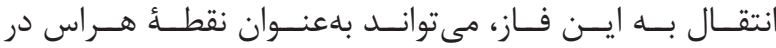

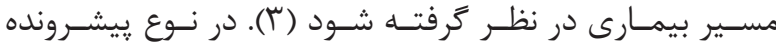

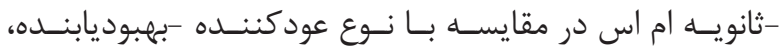

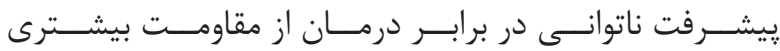

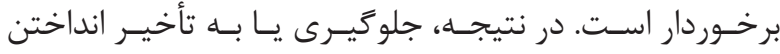

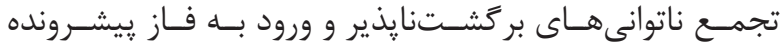

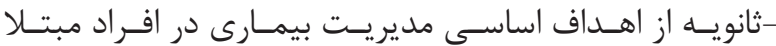

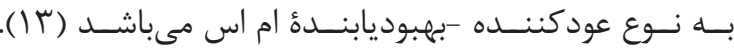

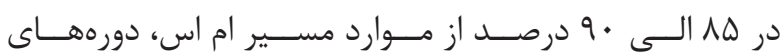

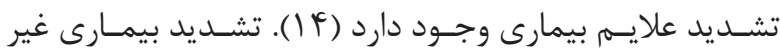

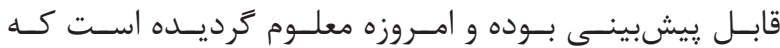

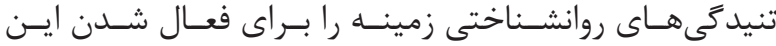

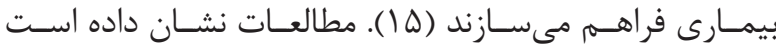

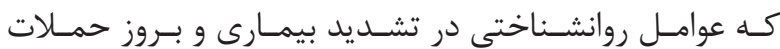

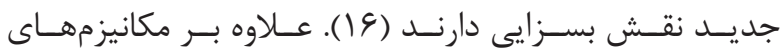

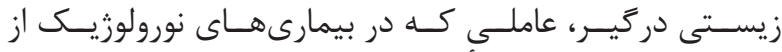

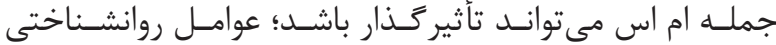

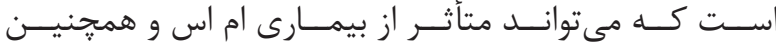

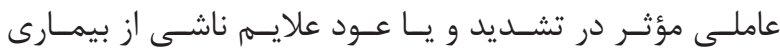

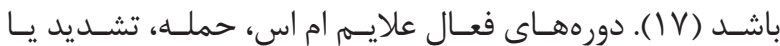
عـود ناميــده مىشــوند (1) (1).

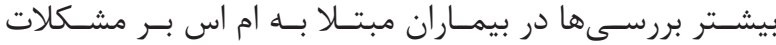

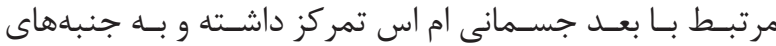

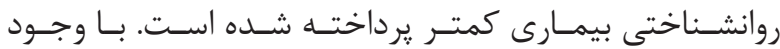

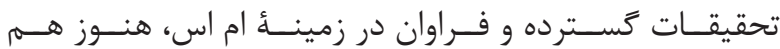

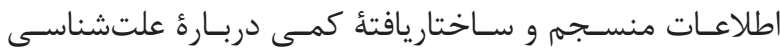

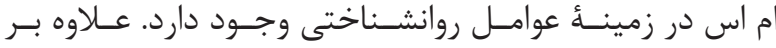

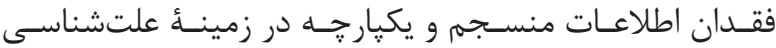

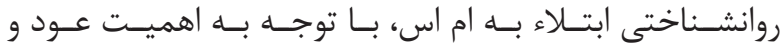

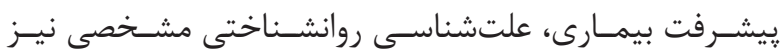

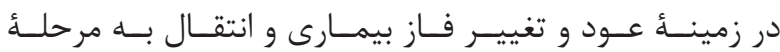

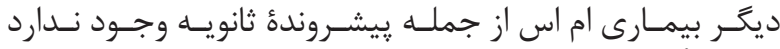

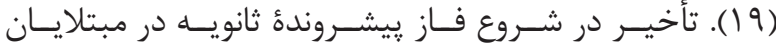

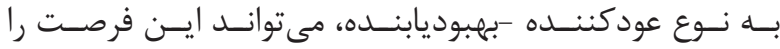

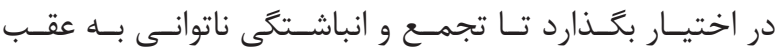

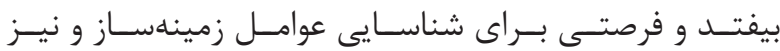

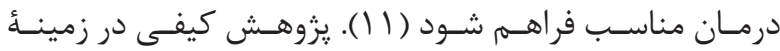

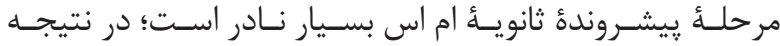

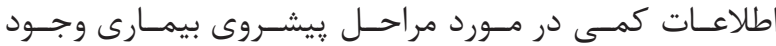

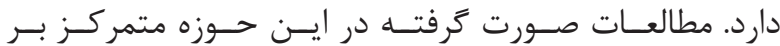

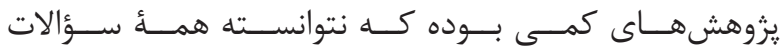

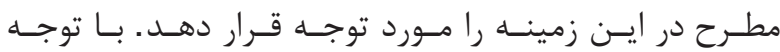

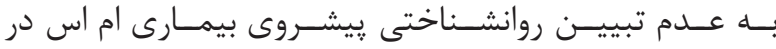

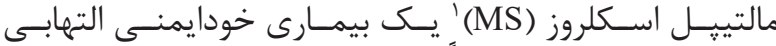

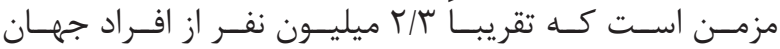

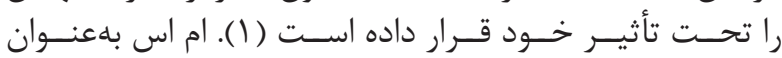

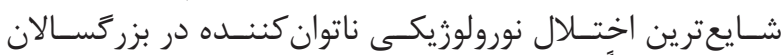

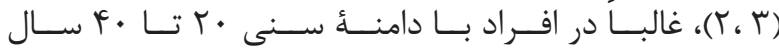

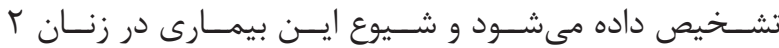

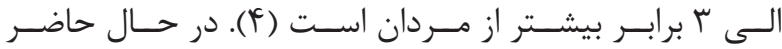

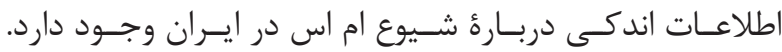

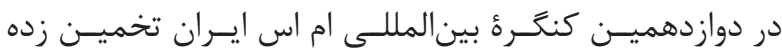

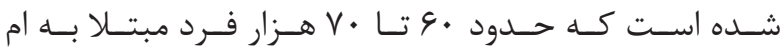

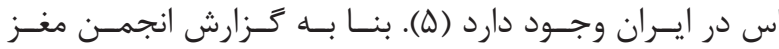

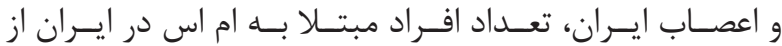

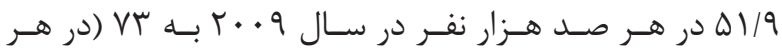

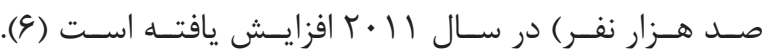

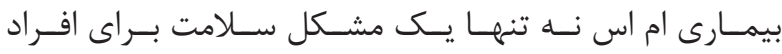

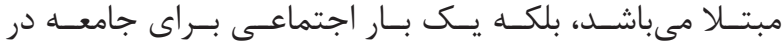

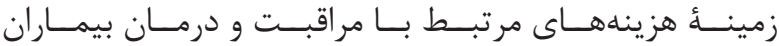

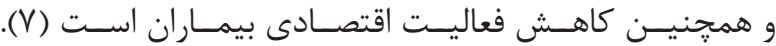

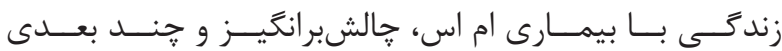

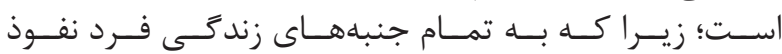

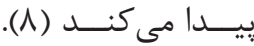

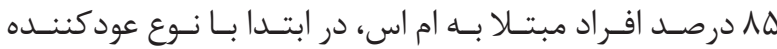

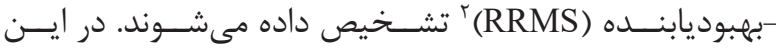

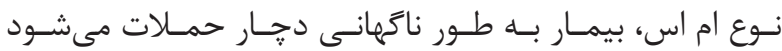

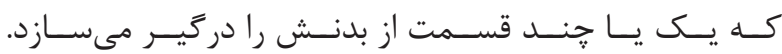

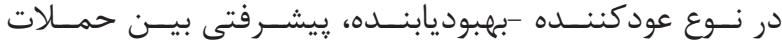

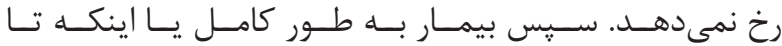

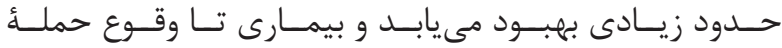

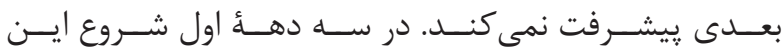

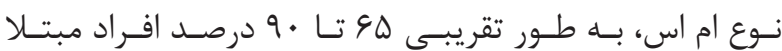

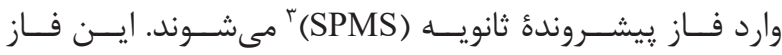

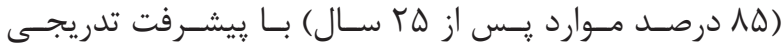

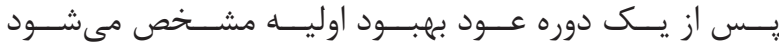

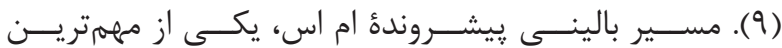

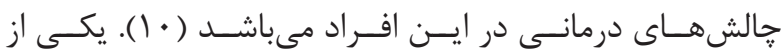

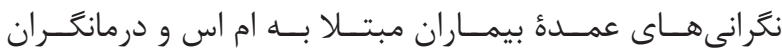

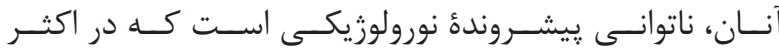
مــوارد اجتنابنايذيــر اســت (1) (1).

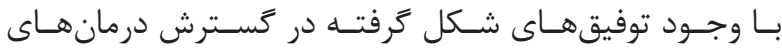

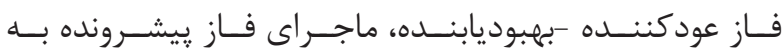

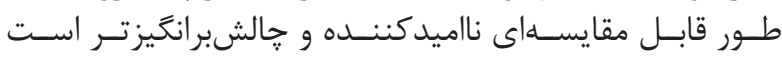

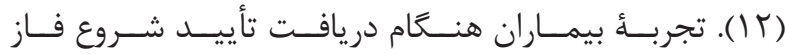




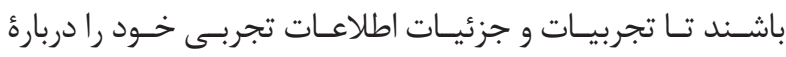

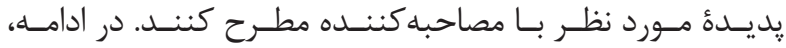

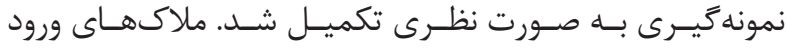

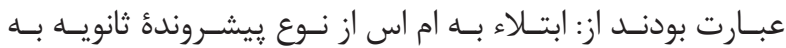

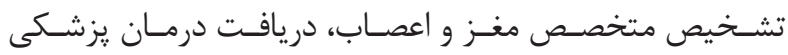

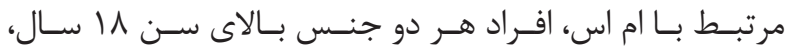

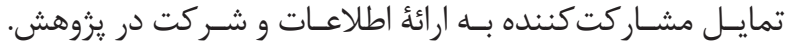

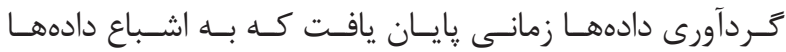

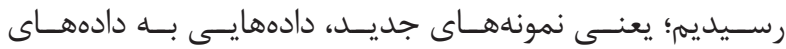

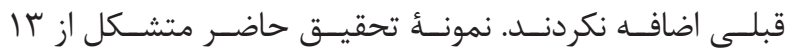

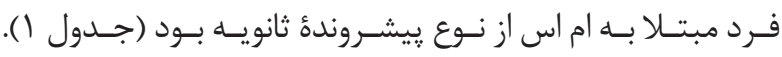

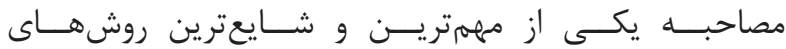

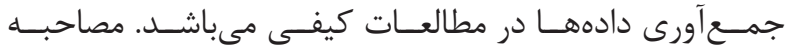

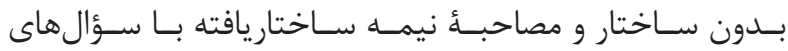

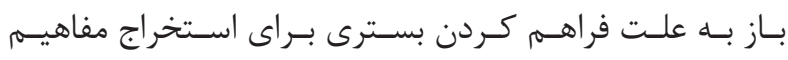

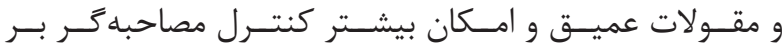

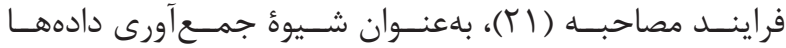

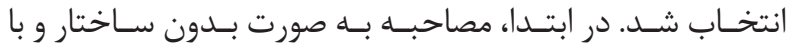

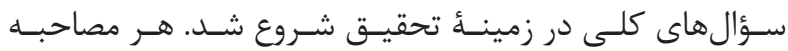

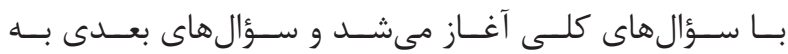

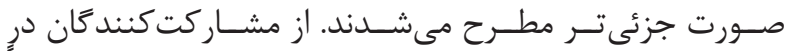

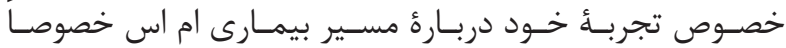

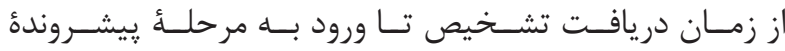

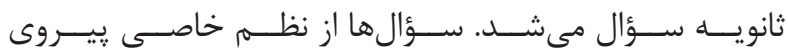

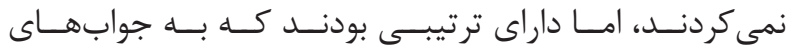

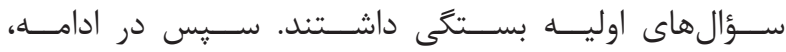

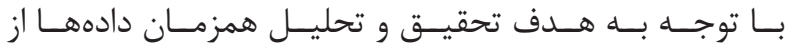

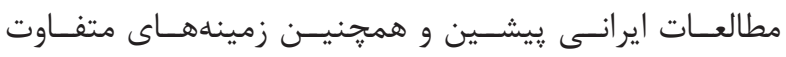

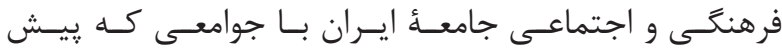

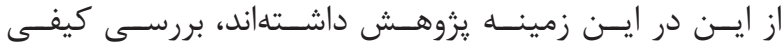

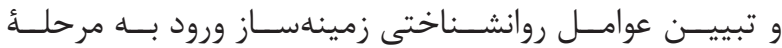

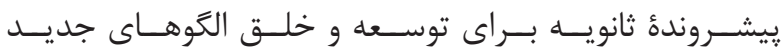

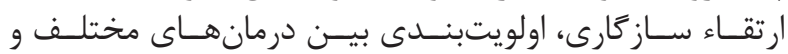

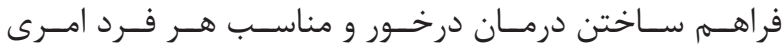

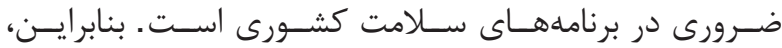

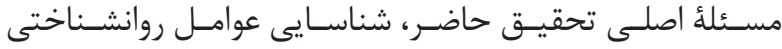

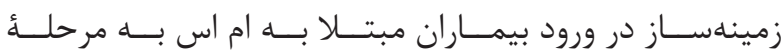

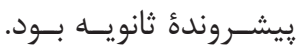

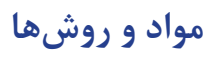

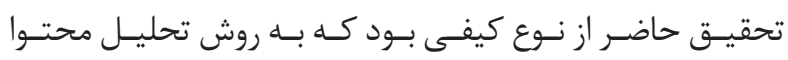

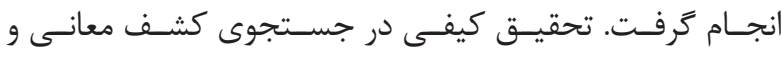

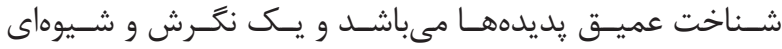

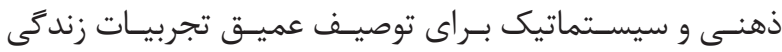

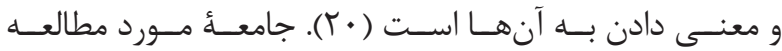

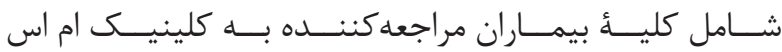

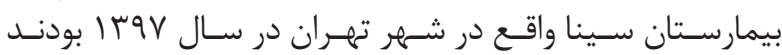

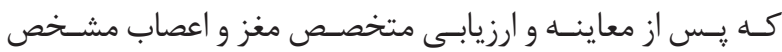

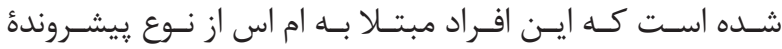

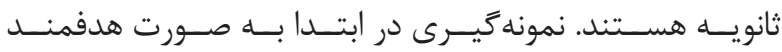

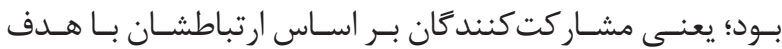

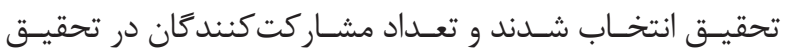

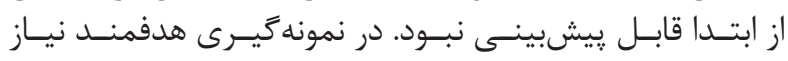

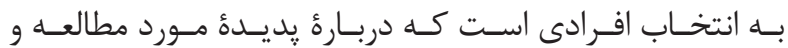

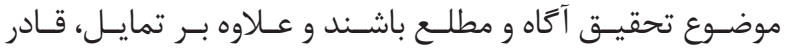

جدول ا- مشخصات مشاركت كندكان.

\begin{tabular}{|c|c|c|c|c|c|c|c|}
\hline SP سال شروع & سال تشخبص RR & شئل & يزئز تحمبلاث & وضعبثت ثأهل & سن & جنسبيت & كد مشاركت ك"نده \\
\hline 91 & $\lambda \xi$ & خاتهدار & دبيلم & متأهل & $\pi$ & مؤنث & lp \\
\hline 91 & $\lambda \xi$ & بيكار & سبكل & متأهل & is & مذكر & $r_{p}$ \\
\hline 91 & $\lambda \varphi$ & شُخل آزاد & ديبلم & هـأهل & pe & مذكر & $\mu_{p}$ \\
\hline ar & AV & خاتهار & سبكل & مئأهل & ps & مؤنث & pp \\
\hline 90 & 91 & بيكار & كارشنالسى & هجرد & r. & مؤنث & $\Delta_{p}$ \\
\hline$\lambda V$ & Vq & بيكار & كارشناسى & هجرد & טז & مؤنث & $e^{p}$ \\
\hline 90 & ye & خاتهدار & دبيلم & متأعل & rq & مؤنث & $v_{p}$ \\
\hline 90 & AT & شغل آزاد & كارشنسنسى & متأعل & il & مذكر & $\Lambda_{p}$ \\
\hline qf & yq & بازنشيـئه & ديبلم & متأهل & f. & مذكر & $q_{p}$ \\
\hline$\lambda \cdot$ & $y_{r}$ & شغل آزاد & فوق ديبلم & متأهل & il & مذكر & 1.8 \\
\hline 98 & $9 \pi$ & خاتهدار & دبيلم & متأهل & TY & مؤُنث & $11 \mathrm{p}$ \\
\hline Qf & Ar & خانهار & سبكل & متأهل & or & مؤنث & ir \\
\hline qr & $\lambda f$ & خاتهار & دبيلم & متأعل & p. & مؤنث & $\pi_{p}$ \\
\hline
\end{tabular}




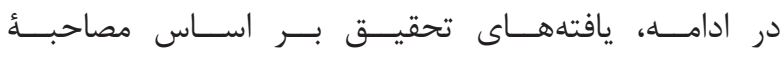

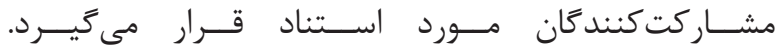

الف) باورهاى مركزى اين مقوله شامل ه زيرمقوله مىباشد. - باور بىارزشى و دوست داشتنى نبودن

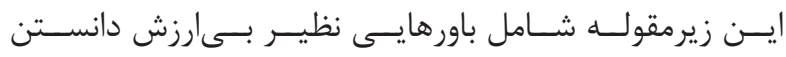

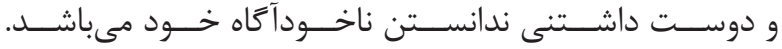

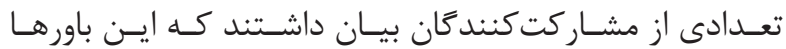

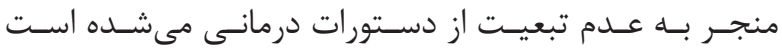

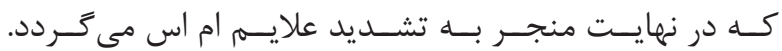

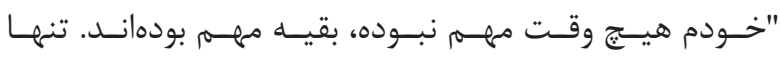

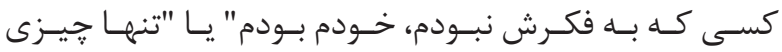

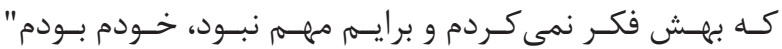

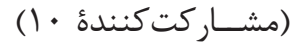

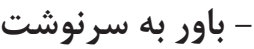

زيرمقولــهُ زمينهســاز شناسـايى شـدهُ ديخــر در ايسن تحقيـق

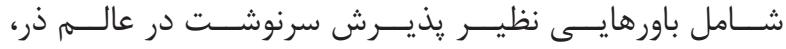

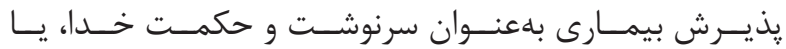

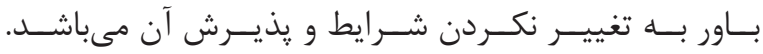

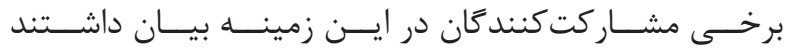

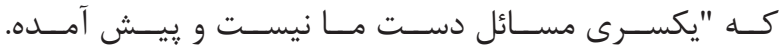

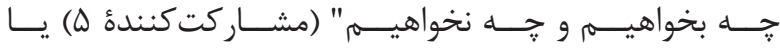

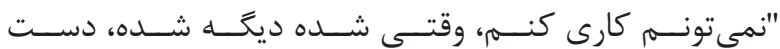

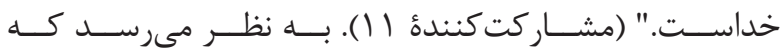

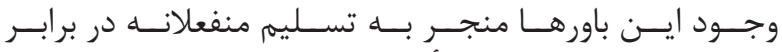

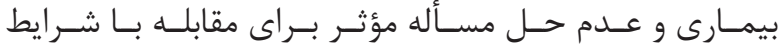

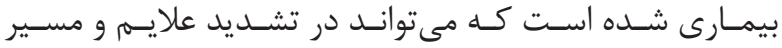

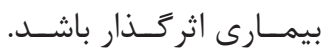

\section{- باور ناامن بودن دنيا}

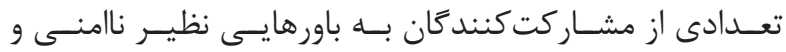

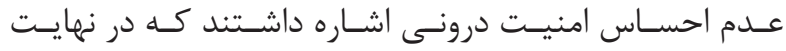

مصاحبــهُ نيمــه ســاختاريافته اسـتفاده شــد. قبــل از شـروع مصاحبــهـ توضيحـات كاملـى بــه زبان كامـلًا غير

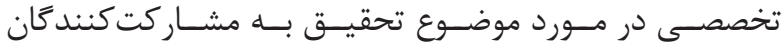

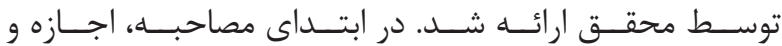

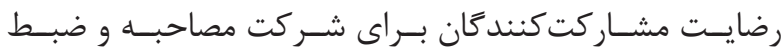

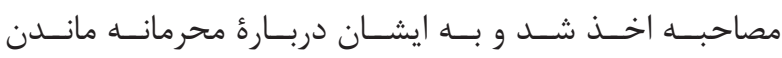

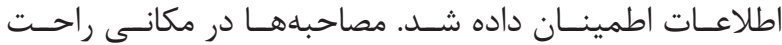

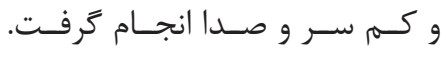

بــراى ارزشــيابى درسـتى، قابليـت اطمينــان و مؤثـق بــودن

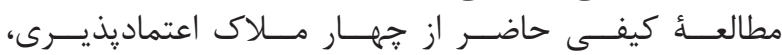

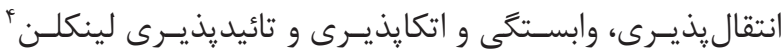

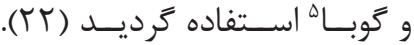

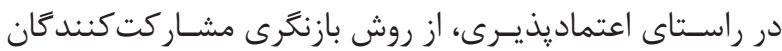

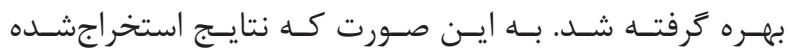

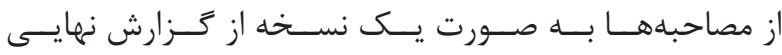

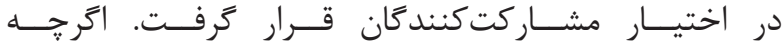

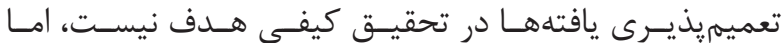

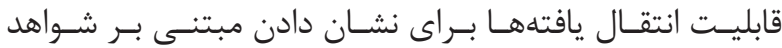

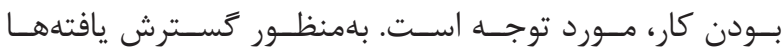

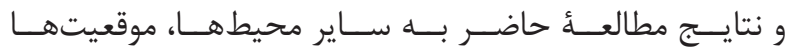

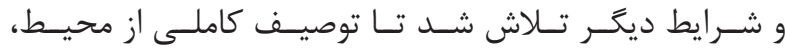

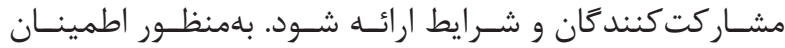

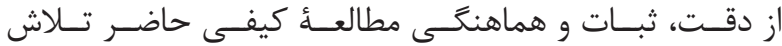

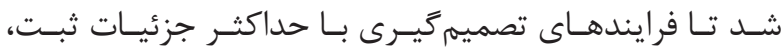

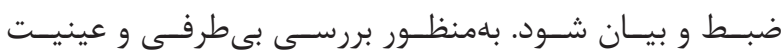

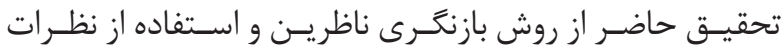

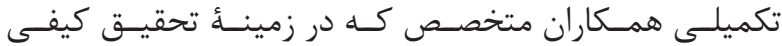

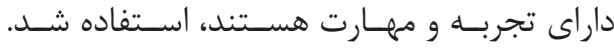

يافته ها

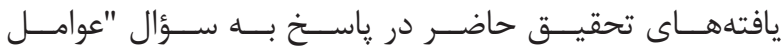

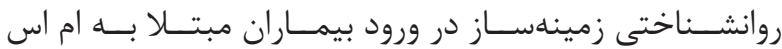

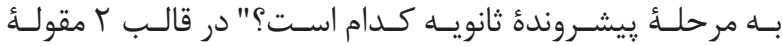

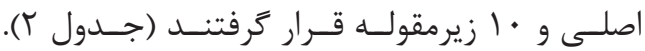

\begin{tabular}{|c|c|c|}
\hline زيرمقوله & مقوله اصلى & مؤلفه \\
\hline 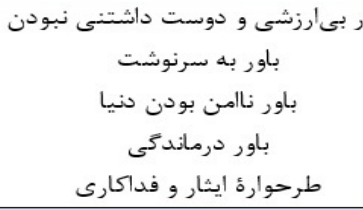 & باو رهاى مركزى & ساز و كارهاى بنـيادين \\
\hline 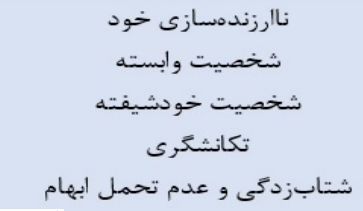 & صفات شخصيتى & \\
\hline
\end{tabular}

${ }^{4}$ Lincoln

${ }^{5} \mathrm{Guba}$ 


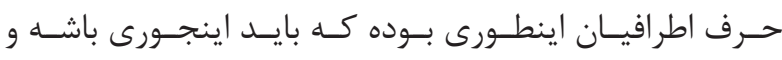

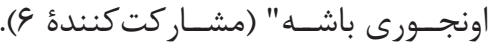

- شخصيت خودشيفته

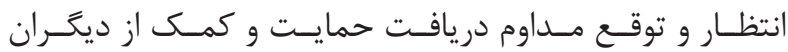

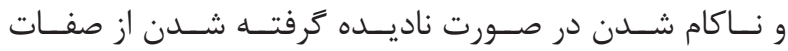

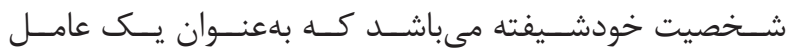

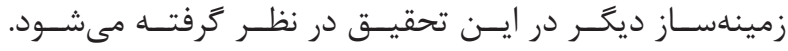

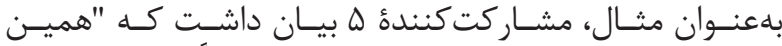

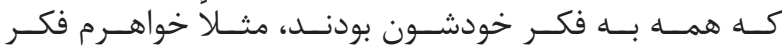

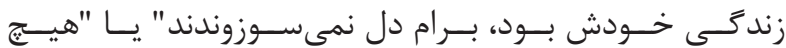

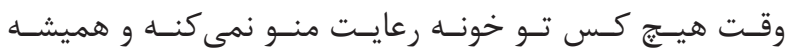

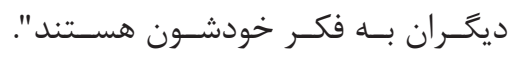

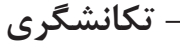

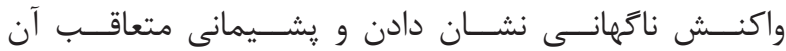

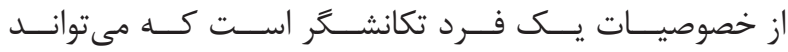

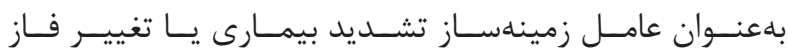

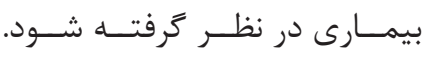

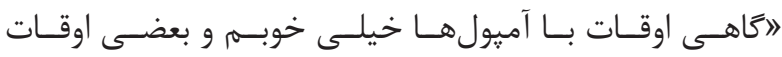

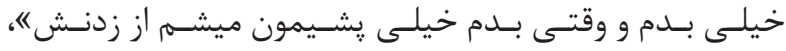

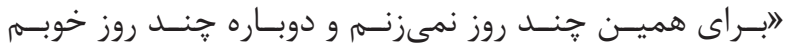

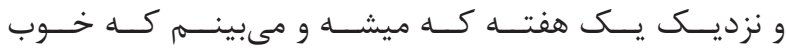

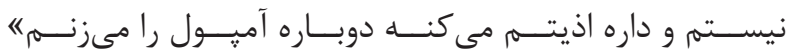

(مشـاركت كنندة سار).

\section{- متابزدכى و عدم تحمل ابهام}

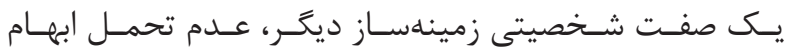

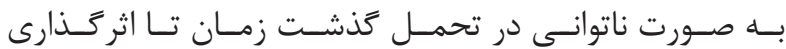

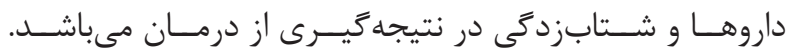

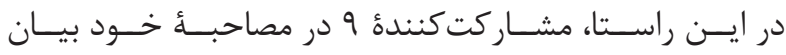

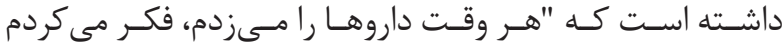

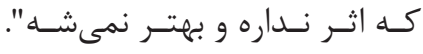

بحث و نتيجه

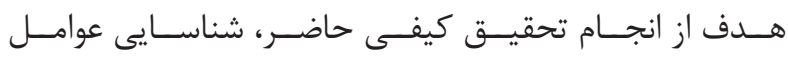

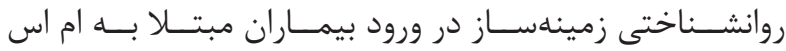

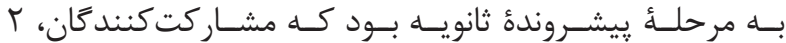

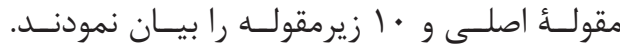

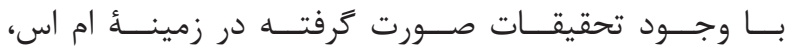

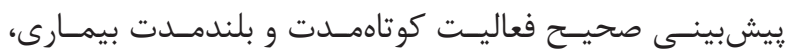

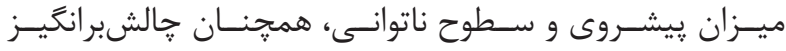

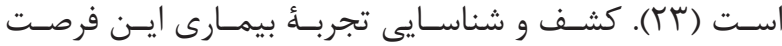

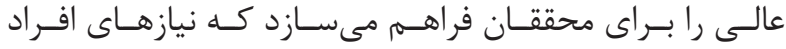

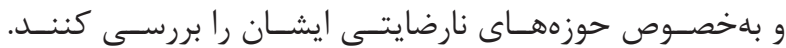

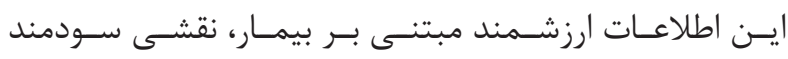

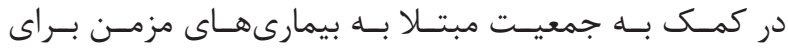

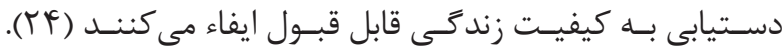

منجــر بــه يـــ احسـاس اضطــراب فراخيــر و مزمـن مىشـود.

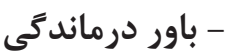

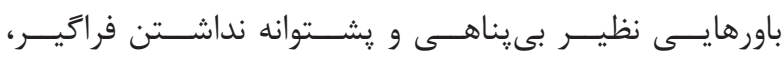

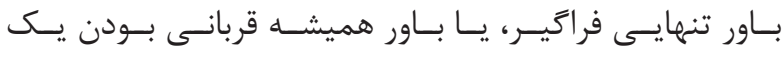

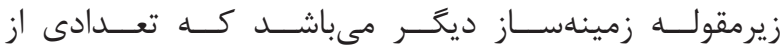

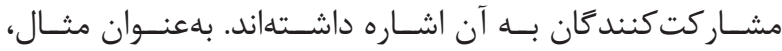

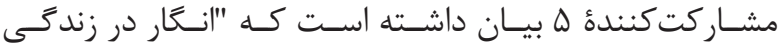

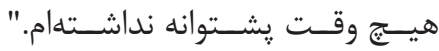

- طرحوارة ايثار و فداكارى - ل

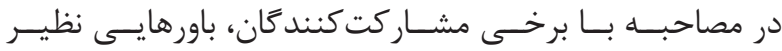

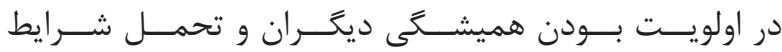

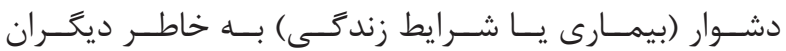

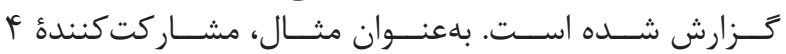

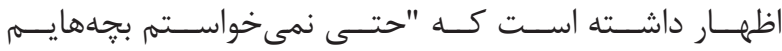

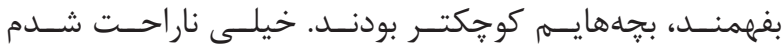

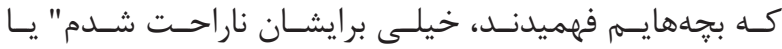

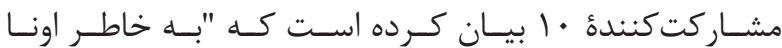

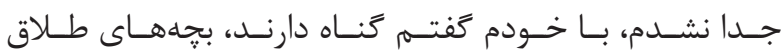

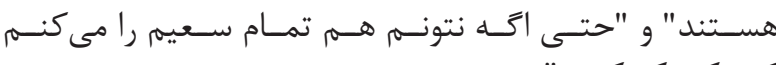

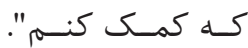

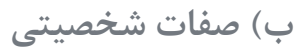

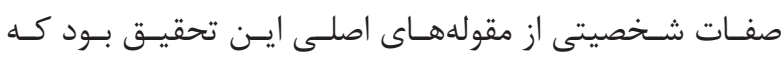

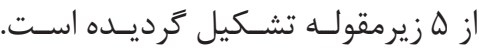

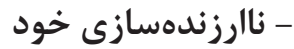

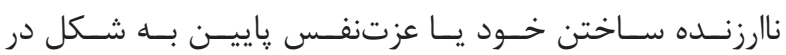

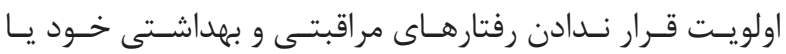

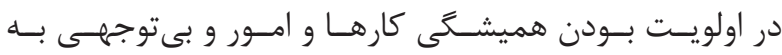

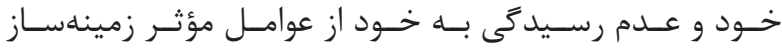

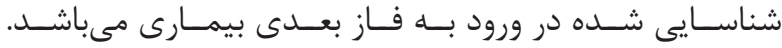

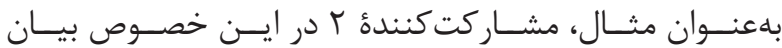

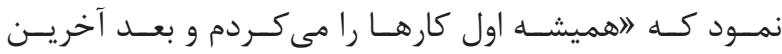

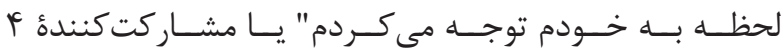

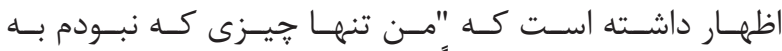

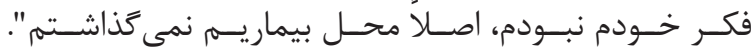

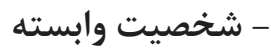

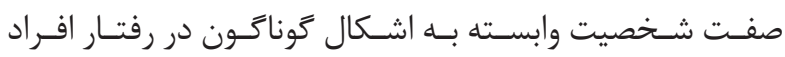

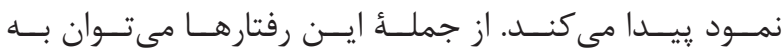

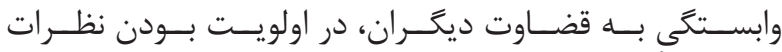

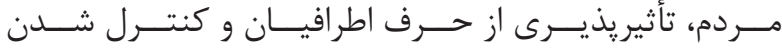

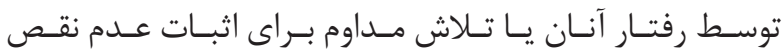

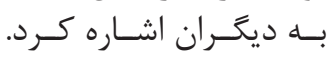

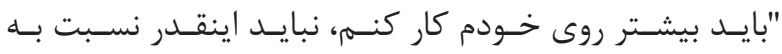

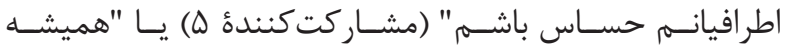


و مرتبــط بـــا ســلامت خويــش و دســتورات درمانــى را در

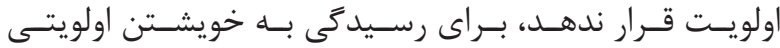

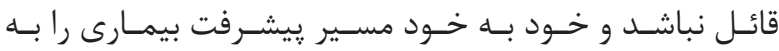
تســريع بينــدازد.

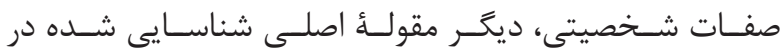

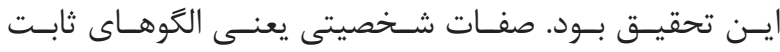

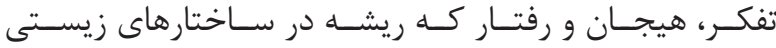

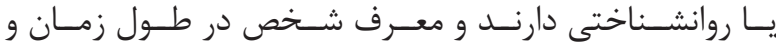

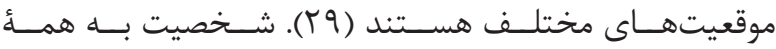

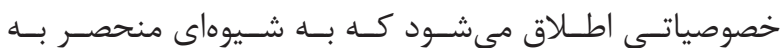

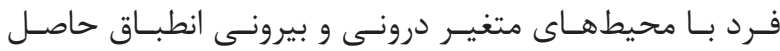

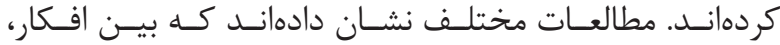

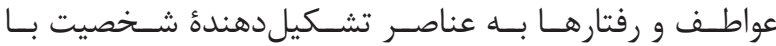

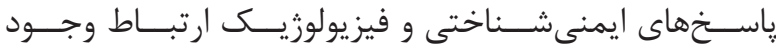

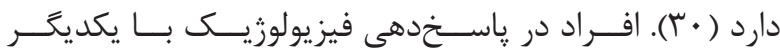

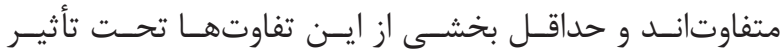

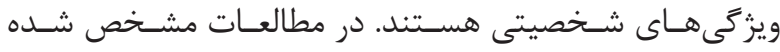

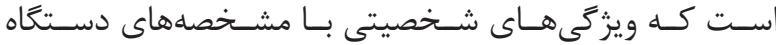

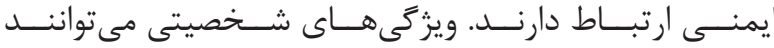

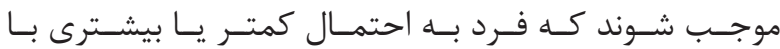

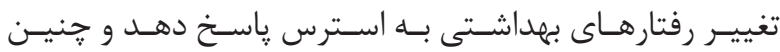

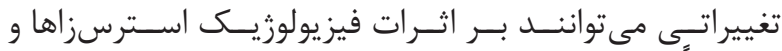

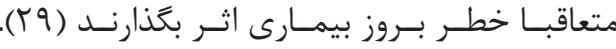

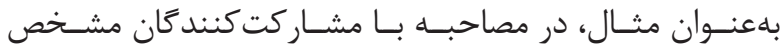

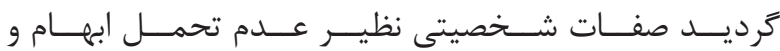

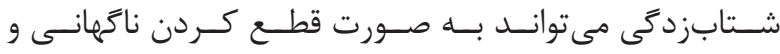

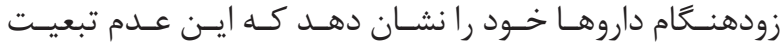

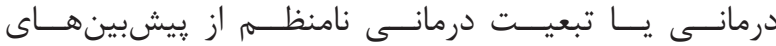

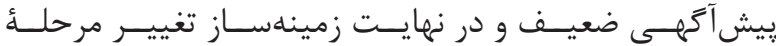

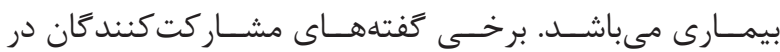

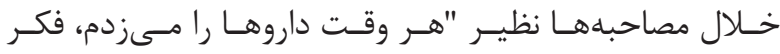

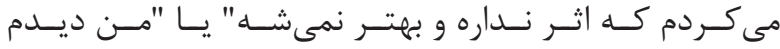

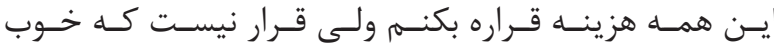

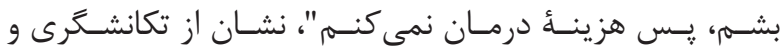

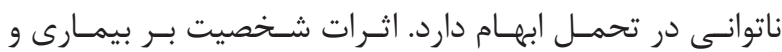

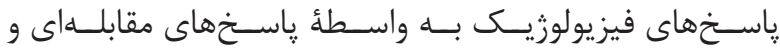

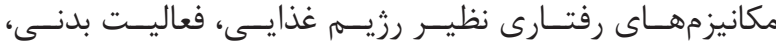

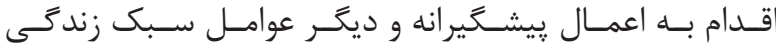

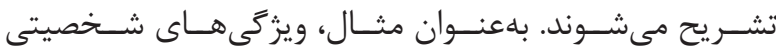

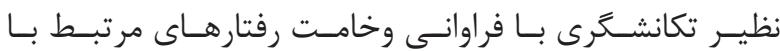

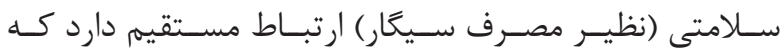

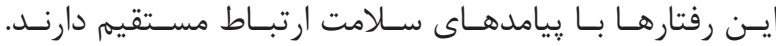

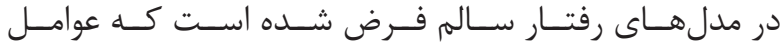

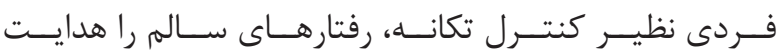

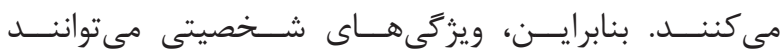

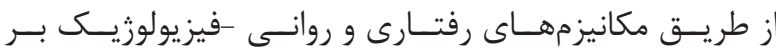

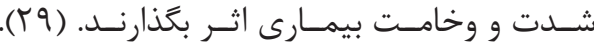

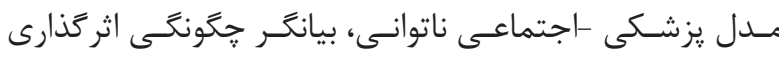

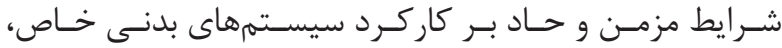

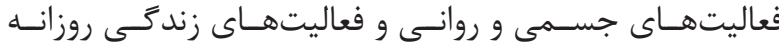

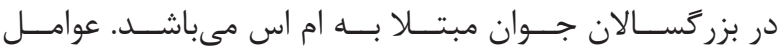

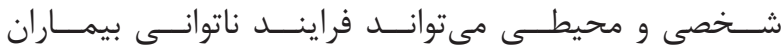

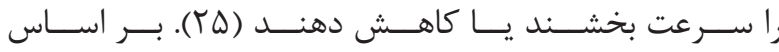

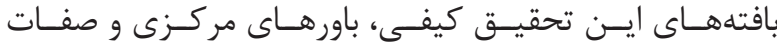

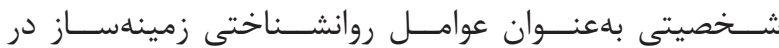

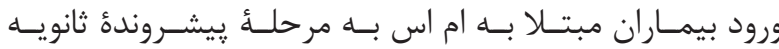

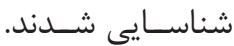

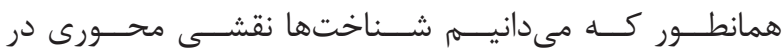

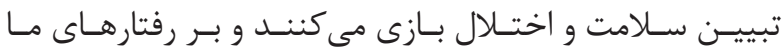

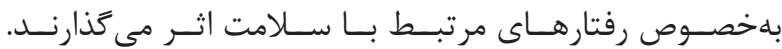

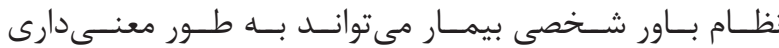

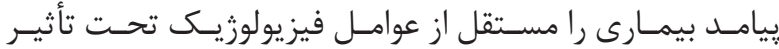

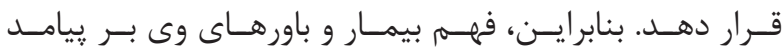

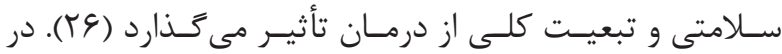

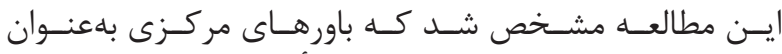

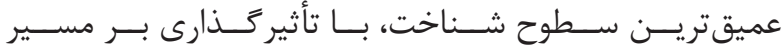

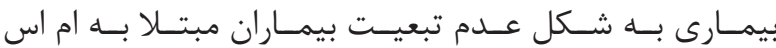

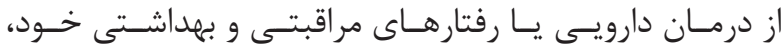

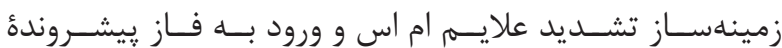

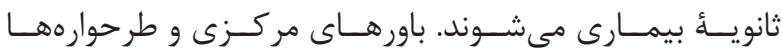

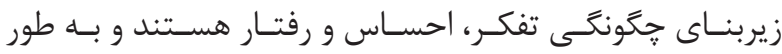

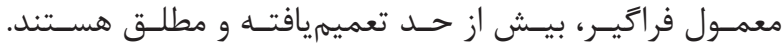

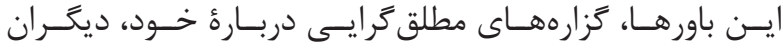

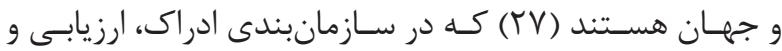

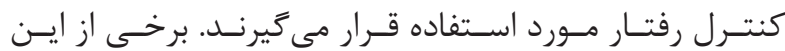

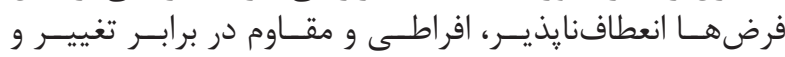

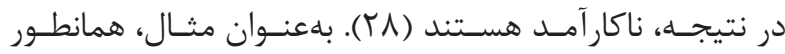

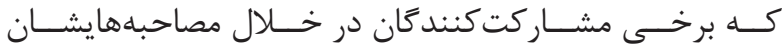

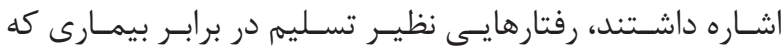

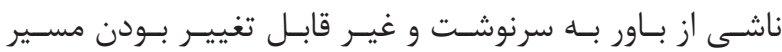

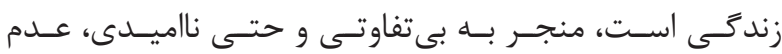

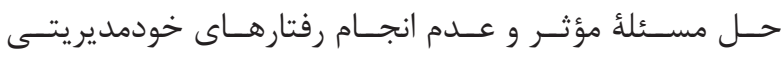

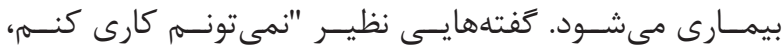

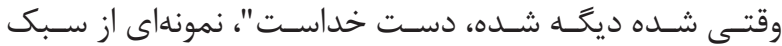

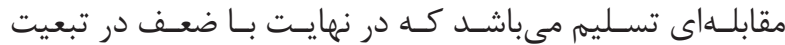

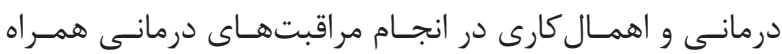

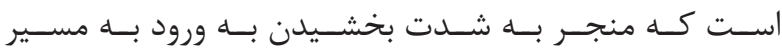

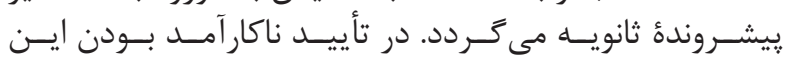

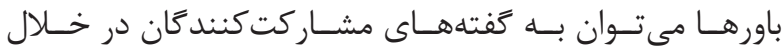

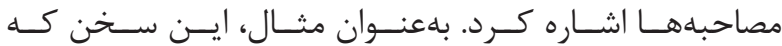

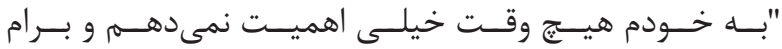

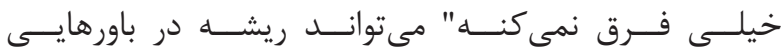

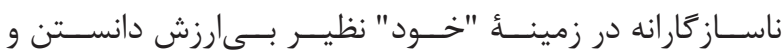

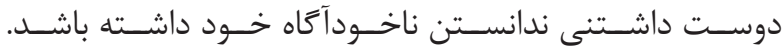

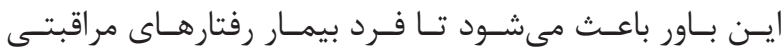




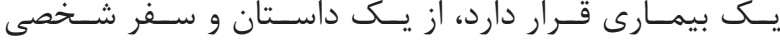

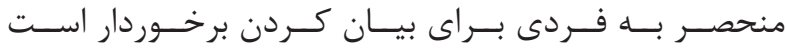

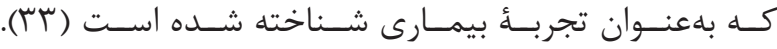

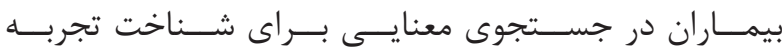

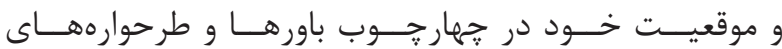

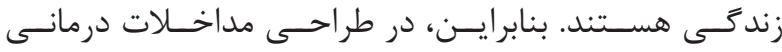

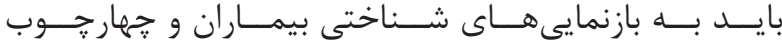

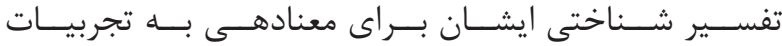

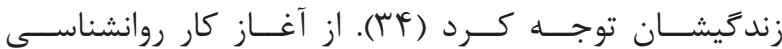

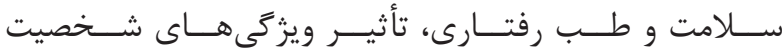

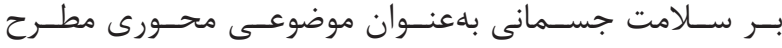

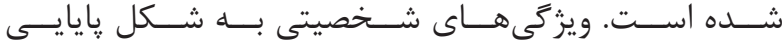

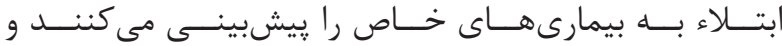

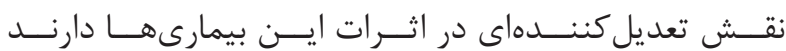

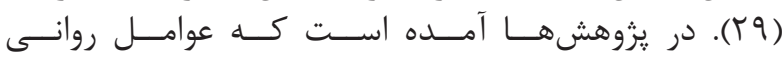

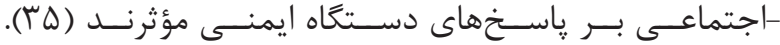

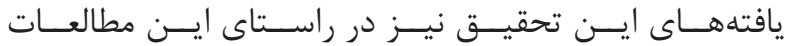

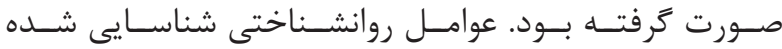

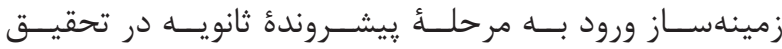

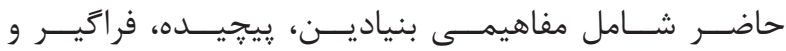

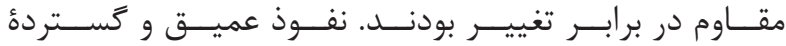

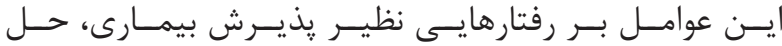

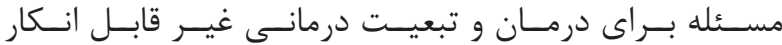

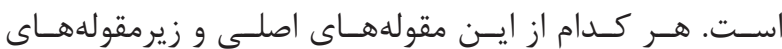

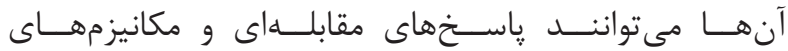

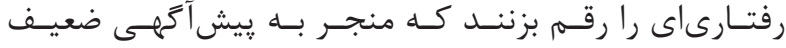

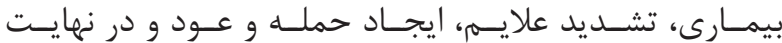

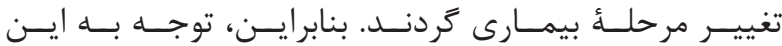

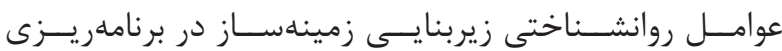

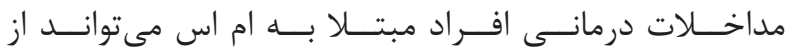

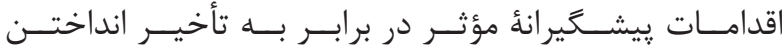

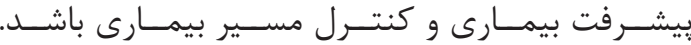

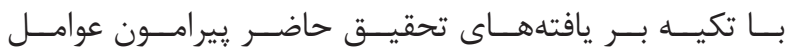

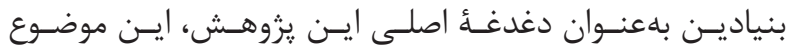

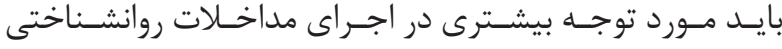

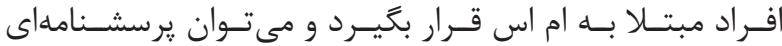

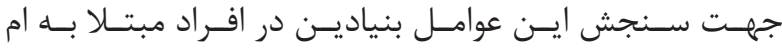

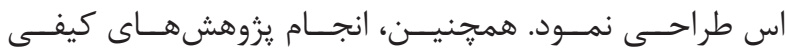

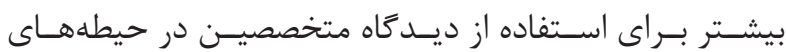

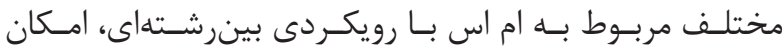

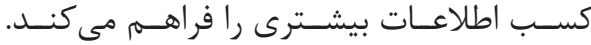

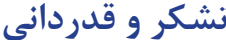

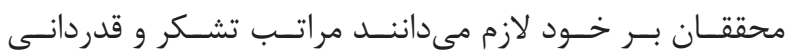

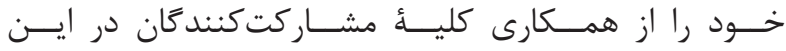

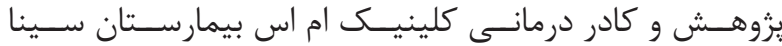

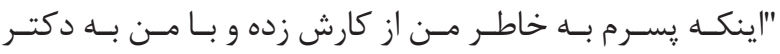

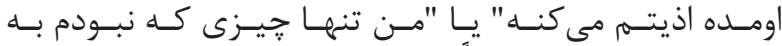

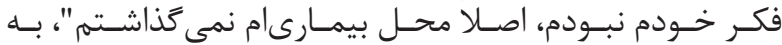

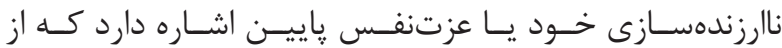

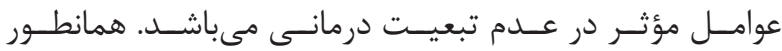

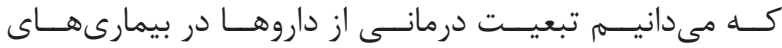

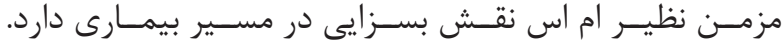

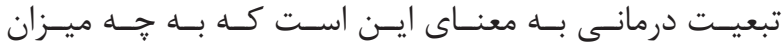

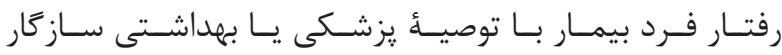

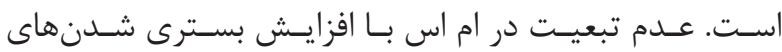

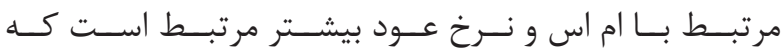

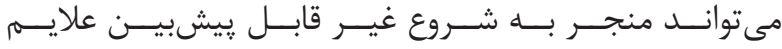

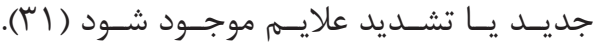

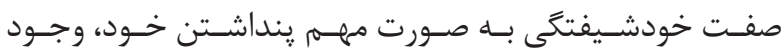

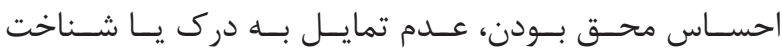

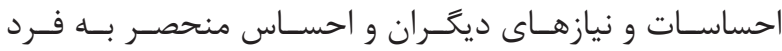

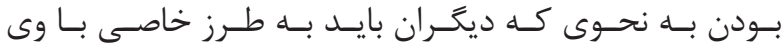

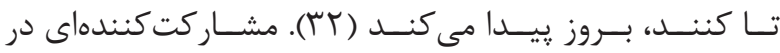

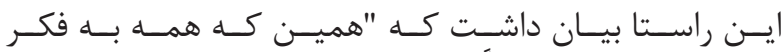

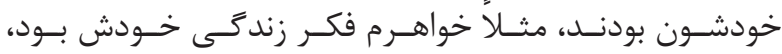

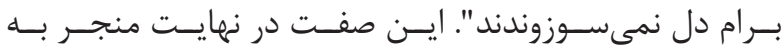

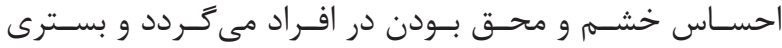

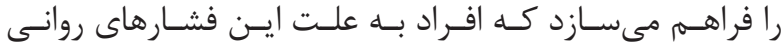

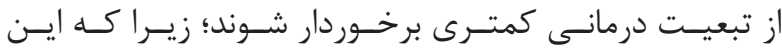

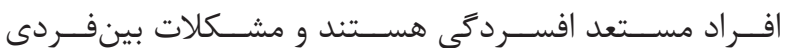
بيشـترى را تجربــه مى كنــــد.

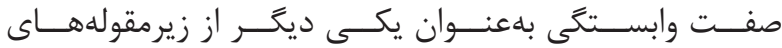

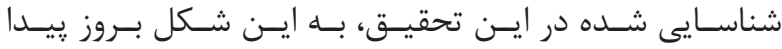

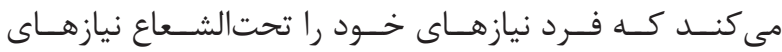

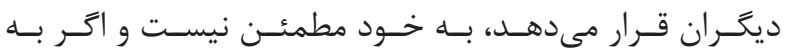

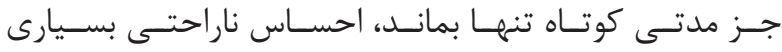

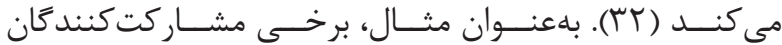

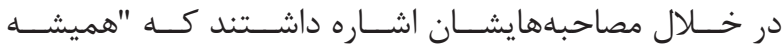

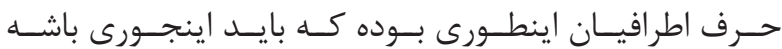

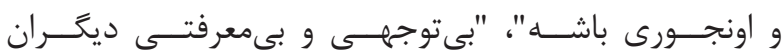

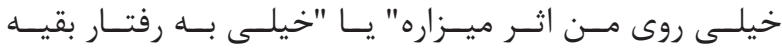

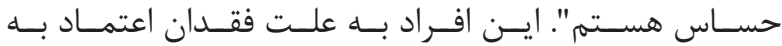

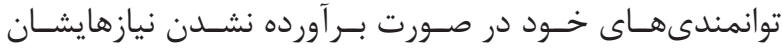

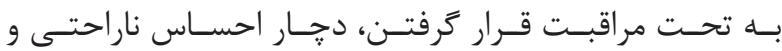

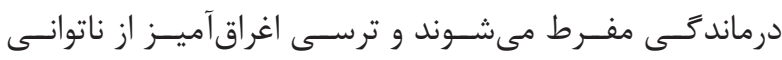

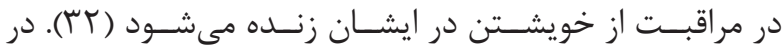

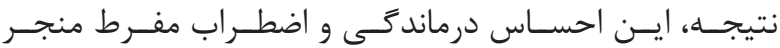

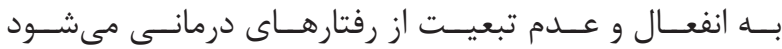

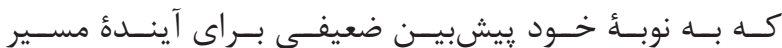
بيمـارى اسـت.

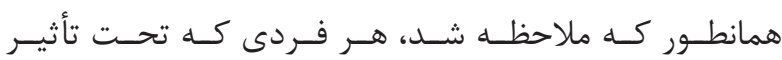


1. Soundy A, Roskell C, Elder T, Collett J, Dawes H. The psychological processes of adaptation and hope in patients with multiple sclerosis: a thematic synthesis Open Journal of Therapy and Rehabilitation. 2016; 4(01): 22.

2. Dennison L, Smith EM, Bradbury K, Galea I. How do people with Multiple Sclerosis experience prognostic uncertainty and prognosis communication? A qualitative study. PloS One. 2016; 11(7): e0158982.

3. Davies F, Edwards A, Brain KA, Edwards M, Jones $\mathrm{R}$, Wallbank R, et al. 'You are just left to get on with it': qualitative study of patient and carer experiences of the transition to secondary progressive Multiple Sclerosis. BMJ. 2015; 5(7): e007674.

4. Heller M, Taylor D. Greater expectations: the future hopes of people with Multiple Sclerosis. UCL School of Pharmacy. 2017; 1-32.

5. Ebrahimi H, Hassankhani H, Khodadadi E, Namdar $\mathrm{H}$, Ferguson C. Experiences of Iranian family caregivers supporting individuals with multiple sclerosis: A qualitative study. International Journal of Medical Research and Health Sciences. 2016; 5(9): 174-82.

6. Ebrahimi H, Hasankhani H, Namdar H, Khodadadi E, Fooladi M. Dealing with chronic illness: experiences of Iranian families of persons with multiple sclerosis-a qualitative study. Mult Scler Int. 2017; 1-8.

7. Milo R, KahanaE. Multiplesclerosis: geoepidemiology, genetics and the environment. Autoimmun Rev. 2010; 9(5): A387-94.

8. Cameron M, Finlayson M, Kesselring J. Multiple sclerosis basics. Multiple sclerosis rehabilitation: From impairment to participation. New York: CRC Press. 2013; p. 9-34.

9. O'Loughlin E. The experience of transitioning from relapsing remitting to secondary progressive Multiple Sclerosis. PhD Thesis. United Kingdom: University of London. 2015.

10. Pandey KS, Krieger SC, Farrell C, Hannigan $\mathrm{C}$, DeAngelis T, Miller AE, et al. Clinical course in multiple sclerosis patients presenting with a history of progressive disease. Multiple Sclerosis and Related Disorders. 2014; 3(1): 67-71.

11. Muris AH, Rolf L, Broen K, Hupperts R, Damoiseaux $\mathrm{J}$, Smolders J. A low vitamin D s tatus at diagnosis is associated with an early conversion to secondary progressive multiple sclerosis. J Steroid Biochem Mol
Biol. 2016; 164: 254-7.

12. Fox RJ, Thompson A, Baker D, Baneke P, Brown D, Browne $\mathrm{P}$, et al. Setting a research agenda for progressive multiple sclerosis: the International Collaborative on Progressive MS. Multiple Sclerosis Journal. 2012; 18(11): 1534-40.

13. Compston A, Coles A. Multiple sclerosis. Lancet. 2015; 372: 1502-17.

14. Liu Y, Morgan C, Hornung L, Tyry T, Salter AR, Agashivala N, et al. Relationship between symptom change, relapse activity and disability progression in multiple sclerosis. J Neurol Sci. 2016; 362: 121-6.

15. Buljevac D, Hop WC, Reedeker W, Janssens AC, Van der Meche FG, Van Doorn PA, et al. Self-reported stressful life events and exacerbations in multiple sclerosis: prospective study. BMJ. 2003; 327(7416): 646.

16. Mir Hashimi M, Najafi F. The effectiveness of solidarity therapy on resilience and cohesion in Multiple Sclerosis (MS) patients. Journal of Medical Sciences of Islamic Azad University. 2014; 24(3): 175-81.

17. Shamili F, Zare H, Oraki M. Predicting the quality of life in patients with Multiple Sclerosis based on disease perception. Orumia Medical Journal. 2013; 24(6): 379-92.

18. Feinstein, A. The clinical neuropsychiatry of multiple sclerosis. New York: Cambridge University Press. 2007.

19. Chruzander C, Johansson S, Johansson K, Einarsson U, Fredrikson S, et al. A 10-year follow-up of a population-based study of people with multiple sclerosis in Stockholm, Sweden: Changes in disability and the value of different factors in predicting disability and mortality. J Neurol Sci. 2013; 332(1-2): 121-7.

20. Hooman HA. Handbook of qualitative research. Tehran: Samt Publications. 2007.

21. Willig C. Introducing qualitative research in psychology: adventures in theory and method. New York: Open University Press. 2008.

22. Corbin JM, Strauss AL. Basics of qualitative research: techniques and procedures for developing grounded theory. $3^{\text {rd }}$ ed. Thousand Oaks, CA: Sage Publications, Inc. 2008 .

23. Dennison L, Smith EM, Bradbury K, Galea I. How do people with Multiple Sclerosis experience prognostic uncertainty and prognosis communication? A qualitative study. PloS One. 2016; 11(7): e0158982. 


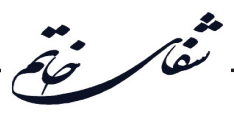

24. Malcomson KS, Lowe-Strong AS, Dunwoody L. What can we learn from the personal insights of individuals living and coping with multiple sclerosis? Disabil Rehabil. 2008; 30(9): 662-74.

25. Krokavcova M, van Dijk JP, Nagyova I, Rosenberger J, Gavelova M, Middel B, et al. Social support as a predictor of perceived health status in patients with multiple sclerosis. Patient Education and Counseling. 2008; 73(1): 159-65.

26. Rahimina Boogar S. Clinical health psychology: prevention, formulation, and treatment guide. Tehran: Danjehpub. 2010.

27. Rostami R, Bidadian M, Bahramizadeh H. Practical cognitive-behavioral depression therapy: clinical education for therapists. Tehran: Arjmand Publications. 2015.

28. Clark DM, Kirk Y, Salcus Case P, Hawton K. Cognitive behavioral therapy: A practical guide to the treatment of mental disorders. Tehran: Arjmand Publications. 1997.

29. Boll TJ. Handbook of clinical health psychology of behavior and health: disorders of behavior and health. Tehran: Arjmand Publications. 2004.

30. Mehrinejad SA, Hosseini Abadi Shapouri M, Khosravani Shariati SH. Comparison of personality characteristics of patients with multiple sclerosis and normal individuals. Psychological Studies. 2012; 8(4): 95-105.

31. Wilkinson HR. Living with unpredictability in Multiple Sclerosis. PhD Thesis. University of Lincoln. 2014.

32. Sadock BJ, Sadock VA, Ruiz P. Kaplan and Sadock's synopsis of psychiatry: behavioral sciences/clinical psychiatry. Tehran: Arjmand Publications. 2014.

33. Langgartner M, Langgartner III, Drlicek M. The patient's journey: Multiple sclerosis. BMJ. 2005; 16(330): $885-8$.

34. White CA. Cognitive behavior therapy for chronic medical problems: a guide to assessment and treatment in practice. Tehran: Arjmand Publications. 2001.

35. Sarafino EP. Health psychology: biopsychosocial interactions. Tehran: Roshd Publications. 2002. 